\title{
Advances in Process Synthesis and Control
}

\section{Lixia Kang ${ }^{1} \cdot$ Yongzhong Liu $^{1}$}

Published online: 14 May 2019

(C) Springer Nature Singapore Pte Ltd. 2019
This special section features four articles recommended from Chinese Process Systems Engineering Annual Meeting 2017 (PSE2017) held in Kunming, China, on 26-28 July 2017. The theme of this special section is "Advances in Process Synthesis and Control." These articles aim to provide theoretical methods and analytical tools in the field where important theoretical and practical innovations are converted into immediate applications to promote positive changes in the process industries. A brief review of these articles is as follows.

Gao et al. (Gao et al. 2018), from Changzhou University, are dedicated to find a more energy-efficient route to separate benzene-toluene mixture through mechanical vapor recompression (MVR) distillation. In their study, three improved systems of Organic Rankine Cycle (ORC) were proposed and compared according to their performances on energy saving and thermal efficiency. The ORC system by exhaust stream regenerative cycle coupled with MVR heat pump distillation process was proven to have great prospects in industrial applications.

Shen and his coworkers (Shen et al. 2018), from Xi'an Jiaotong University, established a superstructure-based method for the synthesis of single-contaminant heat-integrated water networks in refineries, aiming at simultaneous water saving and energy saving. In their study, a mathematical model was presented to achieve the multiple targets according to the specific situations. The water loss in wastewater regeneration recycling and regeneration reuse was also considered to generate a more realistic solution.

Dai and Qiu (Dai and Qiu 2017), from Southwest Petroleum University, proposed a half quantitative alarm

Lixia Kang

1x_kang@mail.xjtu.edu.cn

Yongzhong Liu

yzliu@mail.xjtu.edu.cn

1 Department of Chemical Engineering, Xi'an Jiaotong University, No. 28 Xianning West Road, Xi'an 710049, Shaanxi, People's Republic of China priority analysis method to deal with the abnormal situation in industry where too many alarms were raised at the same time. In their study, the event tree analysis (ETA) was used to assess the risk consequences of the occurrence of an alarm. The industrial application of the proposed method was finally verified via case studies of a chemical reactor system and a crude stabilization unit.

The last article is a cooperation of CHALCO Zhengzhou Non-ferrous Metals Research Institute Co. and University of Chinese Academy of Science (Song et al. 2018). In their study, a dynamic analysis based on ASPEN Plus simulator and Origin software package was conducted to explore the effects of reflux ratio on multi-steady states in the dividing wall column (DWC). This work is expected to provide a theoretical and methodological guidance to engineers and researchers in industrial design and actual operation of DWC.

Acknowledgments All the authors and reviewers are gratefully acknowledged for their most valuable contributions. Special thanks should go to the journal office for the excellent support, in particular to Prof. Dominic C Y Foo and Dr. Frank Franco for managing this special section. This special section would not be presented for readers without their continuous support and unremitting effort.

\section{References}

Gao X, Yin X, Yang S, Yang D (2018) Improved Organic Rankine Cycle System coupled with mechanical vapor recompression distillation for separation of benzene-toluene mixture. Process Integration and Optimization for Sustainability

Shen R, Zhang Y, Ma J (2018) A mathematical programming method for optimizing the single-contaminant regeneration heat-integrated water networks. Process Integration and Optimization for Sustainability

Dai Y, Qiu Y (2017) Risk matrix and event tree based half quantitative alarm priority analysis for alarm systems. Process Integration and Optimization for Sustainability

Song E, Li S, Wang E (2018) Dynamic analysis for the multi-steady states in the dividing wall column. Process Integration and Optimization for Sustainability

Publisher's Note Springer Nature remains neutral with regard to jurisdictional claims in published maps and institutional affiliations. 